SISSA-89/2004/EP

LPTHE-04-31

CPHT-RR063.1104

hep-th/0411249

\title{
New checks and subtleties for AdS/CFT and $a$-maximization
}

\author{
M. Bertolini ${ }^{a}$, F. Bigazzi ${ }^{b, c}$, A. L. Cotrone ${ }^{d}$ \\ $a$ SISSA/ISAS and INFN, Via Beirut 2; I-34014 Trieste, Italy. \\ $b$ LPTHE, Universités Paris VI and VII, 4 place Jussieu; 75005, Paris, France. \\ $c$ INFN, Piazza dei Caprettari, 70; I-00186 Roma, Italy. \\ $d$ CPHT, École Polytechnique, 48 Route de Saclay; F-91128 Palaiseau Cedex, France. \\ bertmat@sissa.it, bigazzi@lpthe.jussieu.fr, Cotrone@cpht.polytechnique.fr
}

\begin{abstract}
We provide a cross-check of AdS/CFT and $a$-charge maximization for a four dimensional $\mathcal{N}=1$ SCFT with irrational $R$-charges. The gauge theory is the low energy effective theory of $N$ D3branes at the tip of the complex cone over the first del Pezzo surface. By carefully taking into account the subtle issue of flavor symmetry breaking at the fixed point, we show, using $a$ maximization, that this theory has in fact irrational central charge and $R$-charges. Our results perfectly match with those inherited from the recently discovered supergravity dual background. Along analogous lines, we make novel predictions for the still unknown AdS dual of the quiver theory for the second del Pezzo surface. This should flow to a SCFT with irrational charges, too. All of our results differ from previous findings in the literature and outline interesting subtleties in a-maximization and AdS/CFT techniques overlooked in the past.
\end{abstract}




\section{Overview}

Placing D3-branes at conical singularities is a useful tool to obtain four dimensional SCFT's with $\mathcal{N}<4$ supersymmetries. If the cone is a Calabi-Yau threefold, the low energy theory on the branes is an $\mathcal{N}=1 \mathrm{SCFT}$. The AdS/CFT correspondence [1] relates the latter to type IIB string theory on $A d S_{5} \times X^{5}$, where $X^{5}$, the base of the cone, is a Sasaki-Einstein (SE) manifold [2, 3]. A deeply explored example is for $X_{5}=T^{1,1}[\underline{3}$ ] which has topology $S^{2} \times S^{3}$. This is a "regular" SE manifold, as it arises as a free $U(1)$ fibration over a Kähler-Einstein space $\left(S^{2} \times S^{2}\right)$. The class of regular SE manifolds is completely known and includes only few other cases. The less trivial ones (their metrics being unknown) arise when the fibration is over a del Pezzo surface $d P_{k}$, obtained as the blow up of $\mathcal{C} \mathcal{P}^{2}$ at $k=3,4,5,6,7,8$ points.

Recently a remarkable result was obtained in 4, where an infinite number of new smooth SE manifolds and their metrics were found. These manifolds were dubbed $Y^{p, q}$, where $p, q$ are co-prime integer labels with $q<p$, 4] (we refer to this paper for any detail on the geometry). Their topology is $S^{2} \times S^{3}$ and they have $S U(2) \times U(1) \times U(1)$ isometry group. These manifolds are either "quasi-regular" (the $U(1)$ fibration is over a space with orbifold singularities) or "irregular" (the Killing vector has non compact orbits). The quasi-regular (resp. irregular) spaces have volumes which are rational (resp. irrational) multipliers of the volume of a unit round $S^{5}$

$$
V\left(Y^{p, q}\right)=\frac{q^{2}\left(2 p+\sqrt{4 p^{2}-3 q^{2}}\right)}{3 p^{2}\left(3 q^{2}-2 p^{2}+p \sqrt{4 p^{2}-3 q^{2}}\right)} \pi^{3} .
$$

AdS/CFT relates $V\left(X_{5}\right)$ to the $a$-central charge ${ }^{1}$

$$
a=\frac{3}{32}\left(3 \operatorname{Tr} R^{3}-\operatorname{Tr} R\right)
$$

of the dual IR SCFT via the holographic relation [1]

$$
V\left(X^{5}\right)=\frac{N^{2}}{4 a\left(X^{5}\right)} \pi^{3},
$$

where $N$ is the number of D3-branes at the tip of the cone. Thus, for the irregular SE manifolds of [4] AdS/CFT predicts that the dual field theories have irrational $a$-charge, and hence irrational $R$-charges at the IR fixed point.

\footnotetext{
${ }^{1}$ Here $\operatorname{Tr} R$ and $\operatorname{Tr} R^{3}$ are the linear and cubic 't Hooft anomalies for the exact $R$-symmetry. Notice that for generic CFT's $a$ differs from the central charge $c=(1 / 32)\left(9 \operatorname{Tr} R^{3}-5 \operatorname{Tr} R\right)$. However, for large $N$ theories having a holographic dual, $\operatorname{Tr} R=0$ and hence $a=c[5$.
} 
In this paper we test this prediction for a particular case of those studied in [4, $Y^{2,1}$, for which, due to recent geometric findings ${ }^{2}$ [] , the dual field theory is known. Indeed $Y^{2,1}$ is shown to be the horizon of the complex cone over the first del Pezzo surface $d P_{1}$. The dual field theory was built for this case years ago (see for instance [7]): it is a quiver theory with gauge group $S U(N)^{4}$, bi-fundamental chiral fields and a superpotential at the IR fixed point. The supergravity prediction for $a$, using Eqs. (1.1), (1.3), is

$$
a\left(Y^{2,1}\right)=N^{2}(-46+13 \sqrt{13})
$$

Another prediction refers to the $R$-charges of baryons in the dual CFT. In fact, D3-branes wrapped on supersymmetric three-cycles $\Sigma_{A}$ in the dual geometry correspond to baryons whose $R$-charge is proportional to the volumes of such three-cycles via the holographic relation 8 , 9 ]

$$
R\left(B_{A}\right)=\frac{\pi}{3} \frac{V\left(\Sigma_{A}\right)}{V\left(X^{5}\right)} N
$$

It is shown in [6] that each SE manifold $Y^{p, q}$ admits two such three-cycles whose volumes, for the case we are interested in, are

$$
V\left(\Sigma_{1}\right)=\frac{1}{36}(7+\sqrt{13}) \pi^{2} \quad, \quad V\left(\Sigma_{2}\right)=\frac{1}{108}(31+7 \sqrt{13}) \pi^{2} .
$$

Hence, using (1.5), we should expect irrational baryon charges in the dual CFT.

Interestingly, for the case at hand the literature offers a purely field theoretical calculation of $a$, [10]. The result was found using the " $a$-maximization prescription" proposed by Intriligator and Wecht (IW) in [10]. This is a remarkable prescription that allows one to determine the exact $R$-charges of a CFT in those cases where these cannot be completely fixed by (super) symmetries or (ABJ) anomaly-vanishing arguments. This happens when the SCFT admits some global symmetry group containing $U(1)$ factors commuting with the non-abelian flavor symmetries and mixing with $U(1)_{R}{ }^{3}$ (this is the case for the theory for $\left.d P_{1}^{4}\right)$. The $a$-maximization prescription states that among the possible choices, the (a priori arbitrary) combination of $U(1)$-charges corresponding to the exact $R$-symmetry is the one which locally maximizes $a$. We will point out that particular care is needed in including, among these $U(1)$ 's, the ones eventually coming from the breaking of non abelian flavor symmetries in the IR. We refer to [10, 11, 12, 13, 14, 15] for more details on $a$-maximization.

\footnotetext{
${ }^{2}$ We thank the authors, D. Martelli and J. Sparks, for having made us aware of their results before their paper was published.

${ }^{3}$ We will refer to $U(1)_{R}$ as one of the possible $R$-symmetries, dubbed $R_{0}$ in [10].

${ }^{4}$ For regular SE manifolds, instead, the symmetries are enough to fix $R$.
} 
The result found in [10] for the quiver theory for the first del Pezzo surface is that $a=(27 / 32) N^{2}$. Similarly, the $R$-charges for the baryons were computed, again predicting rational values [11, 16, 17. At that time this did not produce any puzzle, since AdS computations where based on the (wrong, as the results in [6] have now explicitly shown) assumption that the geometric results would not have been affected by the manifold being an irregular SE manifold. ${ }^{5}$ However, these field theory findings contradict the more recent geometrical predictions (1.4) and (1.6), opening a possible puzzle for AdS/CFT.

In Section 2 we solve the puzzle. Carefully taking into account the flavor symmetry breaking pattern driven by the superpotential (a subtlety which was overlooked in literature) we calculate the $a$-charge and the $R$-charges by using $a$-maximization and find perfect agreement with the results of [6], Eqs. (1.4) and (1.6). This shows that the exact $R$-charges for the theory on $N$ D3-branes at the tip of the complex cone over $d P_{1}$ are indeed irrational. To our knowledge this is the first example of a CFT with irrational $R$-charges having a known holographic dual. Our calculation provides a cross-check of AdS/CFT and $a$-maximization in a (till now) unexplored realm. It also points into refining part of the AdS/CFT techniques for the case of non-regular SE manifolds, as suggested in [6].

In Section 3 we reexamine the SCFT living on $N$ D3-branes on the complex cone over the second del Pezzo surface, $d P_{2}$. This is not Kähler-Einstein, just as $d P_{1}$, and so we expect this case to be subtle, too. The field theory [7] has $S U(N)^{5}$ gauge group and bifundamental chiral matter content. Moreover, there is a superpotential, which crucially breaks all the non-abelian flavor symmetries to abelian subgroups. Assuming that the theory flows to an interacting fixed point and using $a$-maximization, we show that the SCFT at hand has irrational $R$-charges. This is again in contradiction with previous findings [11, 16, 17]. Our results also provide a purely field theoretical prediction on details of the dual (and so far unknown) geometry.

The two main results of this paper can then be summarized as follows:

- From a field theoretical point of view, our findings outline the following simple, but crucial, issue. As remarked in [10, the non-abelian flavor symmetries do not contribute to the exact $R$. However, care is needed in identifying these symmetries in the IR. A superpotential at the IR fixed point, for example, can break some non abelian flavor symmetries to give extra abelian factors. The latter can and do mix with the other $U(1)$ 's and contribute in determining the exact $R$-symmetry.

\footnotetext{
${ }^{5}$ In the regular cases, one can easily prove that the volumes are rational.
} 
- From the AdS/CFT point of view, we provide a non-trivial check for the duality discussed above and a solution of the related puzzle. Moreover, we give a new argument in support of this duality, showing that the symmetries of the supergravity background exactly match the ones in field theory. Finally, our results explicitly stress, as remarked in [6], the peculiarities of AdS/CFT techniques in cases with irregular SE factors.

Now that the AdS/CFT issues for the theory on $d P_{1}$ have been clarified, it would be very interesting to add fractional branes to this system [18], as it was done for the conifold 19, 20. This would break conformal invariance and could reveal interesting dynamics. Work is in progress in this direction [21. Another interesting development would be to study the KK spectrum of type IIB on $A d S_{5} \times Y^{2,1}$, similarly to what has been done for $T^{1,1}$ [22]. This would help in determining the exact bulk field/CFT operator map. Finally, the SCFT duals of general $Y^{p, q}$ have to be uncovered.

\section{The quiver gauge theory for the first del Pezzo}

The quiver gauge theory for $d P_{1}$ was constructed in [7, 23] and is a four dimensional $\mathcal{N}=1$ SCFT with gauge group $S U(N)^{4}$ and bi-fundamental matter. The corresponding

quiver diagram is depicted in Figure 1. Beside the four gauge multiplets, there are also

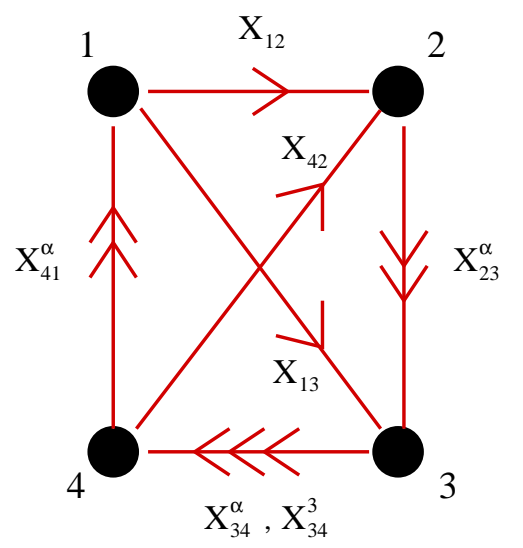

Figure 1: The quiver associated to the first del Pezzo surface. Each dot represents a $S U(N)$ factor while each arrow represents a bi-fundamental chiral multiplet.

ten chiral multiplets $X_{i j}$. The notation is such that the chiral superfield $X_{i j}$ transforms in the $\left(N_{i}, \bar{N}_{j}\right)$. The flavor symmetry group of the theory without superpotential [10] is $S U(3) \times S U(2) \times S U(2) \times U(1) \times U(1)$. 
At the IR fixed point it is believed that the theory has also a superpotential given by

$$
W=\operatorname{Tr}\left[\epsilon_{\alpha \beta} X_{34}^{\alpha} X_{41}^{\beta} X_{13}-\epsilon_{\alpha \beta} X_{34}^{\alpha} X_{42} X_{23}^{\beta}+\epsilon_{\alpha \beta} X_{34}^{3} X_{41}^{\alpha} X_{12} X_{23}^{\beta}\right],
$$

where $\alpha, \beta=1,2$. This explicitly breaks the $S U(3)$ factor (associated to the 3 bifundamental fields $\left.X_{34}^{I}, I=1,2,3\right)$ of the flavor symmetry group to $S U(2) \times U(1)$, as can be seen by the different rôle played by the doublet $X_{34}^{\alpha}$ and the singlet $X_{34}^{3}$ in the above expression. Similarly, only a single diagonal $S U(2)$ survives and a diagonal $U(1)$ of the two abelian factors. Therefore, the residual non- $R$ symmetry group at the IR fixed point is $S U(2) \times U(1) \times U(1)$. Hence, according to what summarized previously, there are two abelian factors which are expected to mix with $U(1)_{R}$ and give the actual exact $R$-symmetry of the IR SCFT.

Note that, as outlined in [10, the theory at hand could be potentially problematic. For two of the nodes in the quiver (3,4 in our notations) the number of fundamental (and anti-fundamental) fields is $N_{f}=3 N_{c}=3 N$. This means that both factors lye at the boundary of the Seiberg conformal window $(3 / 2) N<N_{f}<3 N$ and in particular their one-loop beta function vanishes in the UV. This should imply that both factors are IR free instead of being interacting. We will assume that the whole theory flows to an IR interacting fixed point. We will also assume that the gauge couplings in the IR are sufficiently small to avoid singularities in the $\beta$-functions. The agreement we will find with AdS dual results strengthens these assumptions.

\subsection{Calculating the central charge via $a$-maximization}

Let us discuss the conditions imposed on the $R$-charges by the fact that the theory flows to an IR SCFT with superpotential. We adopt here the same logic as in [13].

Generically, the $\beta$-functions for each quiver factor are proportional to

$$
\beta \sim 3 T(G)-\sum_{A} T\left(r_{A}\right)\left(1-\gamma_{A}\right),
$$

where the scaling dimension of a chiral field $X_{A}$ is $\Delta_{A}=1+\gamma_{A} / 2$, and it is related to the $R$-symmetry charge by $\Delta_{A}=\frac{3}{2} R_{A} . T(G)$ is the Casimir of the adjoint, and $T\left(r_{A}\right)$ the Casimir of the representation $r_{A}$ under which the field $X_{A}$ transforms. For $G=S U(N)$, $T(G)=N$ and $T$ (fund) $=1 / 2$. At the IR interacting fixed point the $\beta$-functions of the four gauge groups should vanish and this gives the conditions (with obvious notations, 
we suppress redundant $S U(2)$ indexes $)^{6}$

$$
\begin{aligned}
& \hat{\beta}_{1 \star} \equiv N+\frac{1}{2} N\left(R_{12}-1\right)+\frac{1}{2} N\left(R_{13}-1\right)+N\left(R_{14}-1\right)=0 \\
& \hat{\beta}_{2 \star} \equiv N+\frac{1}{2} N\left(R_{12}-1\right)+N\left(R_{23}-1\right)+\frac{1}{2} N\left(R_{42}-1\right)=0 \\
& \hat{\beta}_{3 \star} \equiv N+\frac{1}{2} N\left(R_{13}-1\right)+N\left(R_{23}-1\right)+N\left(R_{34}^{(1)}-1\right)+\frac{1}{2} N\left(R_{34}^{(3)}-1\right)=0 \\
& \hat{\beta}_{4 \star} \equiv N+N\left(R_{14}-1\right)+\frac{1}{2} N\left(R_{24}-1\right)+N\left(R_{34}^{(1)}-1\right)+\frac{1}{2} N\left(R_{34}^{(3)}-1\right)=0
\end{aligned}
$$

where $\star$ means "at the fixed point". Following IW's notations, we moved to the double index notation adapted to this quiver theory, $A \rightarrow i j$, and we used the fact that $1-\gamma_{i j}=$ $3\left(1-R_{i j}\right)$ for chiral fields. Moreover, constrained by the $S U(3) \rightarrow S U(2)$ symmetry breaking dictated by the superpotential (2.7) we are taking the still unknown charges $R_{34}^{(3)}$ and $R_{34}^{(1)}=R_{34}^{(2)}$ as different ones. ${ }^{7}$ This point was overlooked in the literature and, as we are going to show, plays a crucial rôle.

Since, in order to compare our results with the string/supergravity predictions, we are interested in the large $N$ limit, we will ignore all terms of order $1 / N^{k}$ with $k>0$. It is easy to verify that in this case

$$
\operatorname{Tr} R \equiv \sum_{\text {ferm }} r(\text { ferm })=N \sum_{i=1}^{4} \hat{\beta}_{i \star}=0
$$

as expected since the theory has a holographic dual. We see that the above condition does not give a new constraint with respect to Eqs. (2.9)-(2.12).

A genuinely new constraint on the $R$-charges comes from the superpotential. Indeed, we have to impose that the superpotential has $R$-charge equal to 2 at the IR fixed point. This gives the conditions

$$
\begin{aligned}
& R_{34}^{(1)}+R_{41}+R_{13}=2, \\
& R_{34}^{(1)}+R_{23}+R_{42}=2, \\
& R_{34}^{(3)}+R_{12}+R_{41}+R_{23}=2 .
\end{aligned}
$$

The conditions (2.9)-(2.12) and (2.14)-(2.16) leave us with only two unknowns to be determined by $a$-maximization. These precisely correspond to the two $U(1)$ factors of

\footnotetext{
${ }^{6}$ These conditions are equivalent to the ABJ anomaly vanishing conditions.

${ }^{7}$ Our choices of the $R$-charges could be rendered more clear by redefining the fields in such a way that $W$ becomes manifestly invariant under the diagonal $S U(2)$. One can show that this would produce the same results we find here.
} 
the global symmetry group which mix with $U(1)_{R}$ to give the exact $R$-symmetry in the IR. We have

$$
\begin{aligned}
R_{13}=R_{42}=R_{34}^{(3)} \equiv x & , \quad R_{14}=R_{23} \equiv y, \\
R_{34}^{(1)}=R_{34}^{(2)}=2-x-y & , \quad R_{12}=2-x-2 y .
\end{aligned}
$$

Using the above values, we find for the "trial" (i.e. the one before maximization), $a$-charge $a_{t}$ (1.2) the $(x, y)$-dependent expression

$$
a_{t}(x, y)=\frac{9 N^{2}}{32}\left[4+4(y-1)^{3}+3(x-1)^{3}+(1-x-2 y)^{3}+2(1-x-y)^{3}\right] .
$$

Extremizing with respect to $x$ and $y$ we find that the maximum for $a_{t}$ is at

$$
x_{m}=-3+\sqrt{13}, y_{m}=\frac{4}{3}(4-\sqrt{13}),
$$

giving an irrational central charge

$$
c=a=a_{t}(\max )=N^{2}(-46+13 \sqrt{13}) .
$$

It is a simple exercise to show that without implementing the flavor symmetry breaking pattern discussed above, hence taking $R_{34}^{1}=R_{34}^{2}=R_{34}^{3}$, the conditions on the vanishing $\beta$-functions and on the $R$-charge of the superpotential leave only one $R$-charge to be determined. Using $a$-maximization the value one would find is rational, $a=27 N^{2} / 32$. The same happens for the $R$-charges.

A technical remark. As pointed out in [10], the $a$-charge "knows" about the unbroken non-abelian flavor symmetries, and the fact that they do not contribute to the exact $R$-charge. So, for example, if one considers the theory above without the superpotential (2.7), there are only two $U(1)$ 's that can mix with $U(1)_{R}$. However, if one performs the $a$-maximization keeping different $R$-charges for all the ten chiral multiplets, one obtains, after imposing the vanishing of the $\beta$-functions, a six-parameter dependent $a_{t}$. Nevertheless, its actual maximum $a_{t}(\max )$ and the $R$-charges turn out to be the correct ones calculated in [10] for the case $W=0$. The same conclusions applies to the $W \neq 0$ theory above.

Therefore, the final lesson is that whenever it is hard to understand which symmetries are preserved in the IR, one can drop this complication and just keep all the $R$-charges different, being guaranteed that anyway $a_{t}(\max )$ will be the correct one. As our calculation shows, the important point is not to over-constrain the $R$-charges, as this obviously affects the determination of the correct maximum of $a_{t}$. 


\subsection{The $R$-charges}

Plugging the solution (2.19) into the relations (2.17) we get the $R$-charges for the bifundamental fields of the theory. These are reported in Table 1.

\begin{tabular}{|c|c|}
\hline$X_{i j}$ & $R_{i j}$ \\
\hline$X_{12}$ & $\frac{1}{3}(-17+5 \sqrt{13})$ \\
$X_{23}^{\alpha}, X_{41}^{\alpha}$ & $\frac{4}{3}(4-\sqrt{13})$ \\
$X_{34}^{\alpha}$ & $\frac{1}{3}(-1+\sqrt{13})$ \\
$X_{34}^{3}, X_{13}, X_{42}$ & $-3+\sqrt{13}$ \\
\hline
\end{tabular}

Table 1: The exact $R$-charges of the bi-fundamental chiral fields for the $d P_{1}$ dual.

It can be checked that with the above assignment the theory does not violate the unitarity bound. For the gauge invariant mesons not entering the superpotential, $X_{34}^{3} X_{41}^{\beta} X_{13}$, $X_{34}^{3} X_{42} X_{23}^{\beta}$ and $X_{34}^{\gamma} X_{41}^{\alpha} X_{12} X_{23}^{\beta}$, this is immediately verifiable, being their conformal dimension $\Delta>1$.

The baryons are color singlets defined from the corresponding bi-fundamental chiral fields as

$$
B_{i j}=\operatorname{det}_{N \times N}\left(X_{i j}\right) .
$$

Hence they do not give any problem to the unitarity bound: their $R$-charge goes like $N$, since $R\left(B_{i j}\right)=N R_{i j}$.

\subsection{A check for AdS/CFT}

In [6] it is argued that the field theory analyzed above should be dual to type IIB string theory on $A d S_{5} \times Y^{2,1}$. As we are going to show, our results indeed confirm this prediction and thus provide the first example of gauge/gravity duality for SCFT's with irrational $R$ charges.

The first important check concerns the central charge. The irrational $a$-charge we have obtained, Eq. (2.20), is in perfect agreement with the supergravity prediction [6], Eq. (1.4) ${ }^{8}$

\footnotetext{
${ }^{8} \mathrm{An}$ analogous striking test of AdS/CFT was obtained in the regular case of the conifold [24]. In that case, however, no $a$-maximization is required to fix the $R$-charges.
} 
Another matching is provided by baryon $R$-charges: using the holographic relations (1.3) and (1.5), we get for the dual volumes of the corresponding three-cycles

$$
V\left(\Sigma_{A}\right)=\frac{3}{4} N \pi^{2} \frac{1}{a} R\left(B_{A}\right)=\left\{\begin{array}{cl}
\pi^{2}(7+\sqrt{13}) / 36 & A=(12) \\
\pi^{2}(31+7 \sqrt{13}) / 108 & A=(34),(13),(42)
\end{array}\right.
$$

which is nothing but Eq. (1.6), that is what computed for the dual geometry in [6].

Finally, let us add another new piece of evidence in support of the duality, showing that also the global symmetries match. The field theory global symmetries are the exact $R$-symmetry just computed and the global $S U(2) \times U(1) \times U(1)$. The $Y^{2,1}$ metric only admits a $S U(2) \times U(1) \times U(1)$ isometry [4. However, the whole supergravity background contains also a non-trivial flux of the self dual RR five-form field strength $F_{5}=d C_{4}$. The KK reduction of $C_{4}$ on the unique independent three-cycle of $Y^{2,1}$ (whose topology is in fact $S^{2} \times S^{3}$ ) provides an additional $U(1)_{B}$ baryonic symmetry. Hence the continuous global symmetry group of the dual field theory is expected to be

$$
S U(2) \times U(1) \times U(1) \times U(1)_{B}
$$

This precisely agrees with the symmetries of the field theory above. One of the three $U(1)$ 's in the latter is thus mapped to $U(1)_{B}$. Let us notice that the field theory at hand is a chiral quiver. Then the baryonic $U(1)_{B}$ do mix with $U(1)_{R}$ to give the exact $R$-symmetry, as remarked in [11. This is different to what happens in non-chiral theories where baryonic symmetries commute with $R\left[10 .{ }^{9}\right.$

\section{The SCFT for the second del Pezzo surface and a prediction for its AdS dual}

The second del Pezzo surface $d P_{2}$ shares with $d P_{1}$ the property of not admitting a KählerEinstein metric [25]. The base of the complex cone over $d P_{2}$ is thus a non-regular SE manifold and its volume is not guaranteed to be rational [26]. Therefore, one could not exclude to get a dual SCFT with irrational $R$-charges by considering D3-branes at the tip of that cone. The dual gauge theory was worked out in [7, 23] and we can now repeat the same rationale we pursued for the case of $d P_{1}$. In this case neither we know the AdS dual, nor a metric for $d P_{2}$. So, our results should furnish novel predictions on the dual geometry.

\footnotetext{
${ }^{9}$ We are grateful to Ken Intriligator for a crucial observation on this point.
} 
There are two known phases for the field theory duals, related by Seiberg duality. The corresponding quivers are shown in Figure 2. Both of them have gauge group $S U(N)^{5}$,
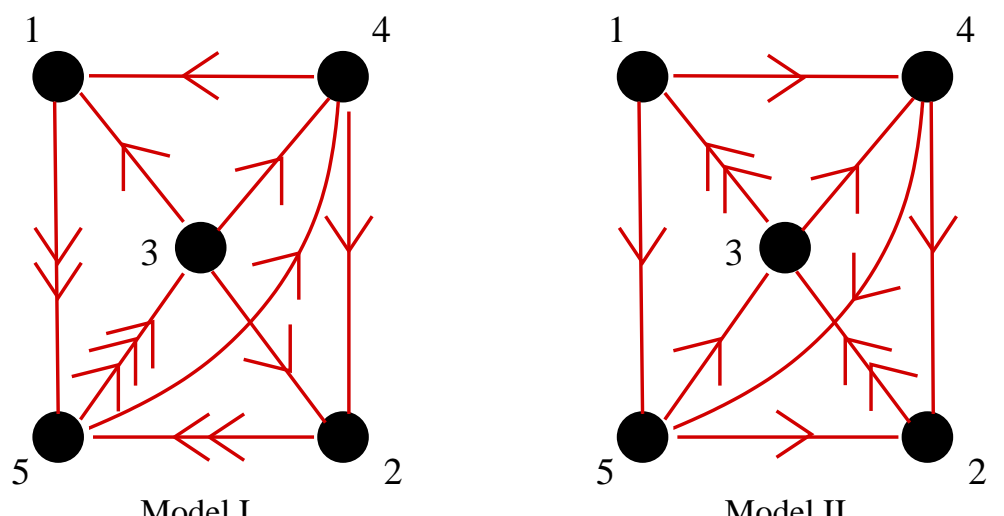

Figure 2: The quivers associated to the second del Pezzo surface.

but different bi-fundamental field content.

Let us first focus on Model II. ${ }^{10}$ In this model all the bi-fundamental fields are flavor singlets but $X_{31}^{\alpha}$ and $X_{23}^{\alpha}(\alpha=1,2)$ which transform in two distinct $S U(2)$ 's. The crucial point is that, again, the superpotential

$$
\begin{aligned}
W= & \operatorname{Tr}\left[X_{34} X_{45} X_{53}-X_{53} X_{31}^{2} X_{15}+X_{34} X_{42} X_{23}^{2}+\right. \\
& \left.+X_{23}^{2} X_{31}^{1} X_{15} X_{52}+X_{42} X_{23}^{1} X_{31}^{2} X_{14}-X_{23}^{1} X_{31}^{1} X_{14} X_{45} X_{52}\right]
\end{aligned}
$$

breaks this non-abelian flavor symmetry. As a result, the $R$-charges of all the bifundamental fields are a-priori different. ${ }^{11}$ We will now compute such charges and the central charge, as in Section 2. By imposing the vanishing of the $\beta$-functions at the IR fixed point and the condition that the superpotential should have $R$-charge 2 , we get four independent $R$-charges, in terms of which we can write

$$
\begin{aligned}
R_{45} \equiv x \quad, \quad R_{53} \equiv y \quad, \quad R_{52} \equiv z \quad, \quad R_{23}^{(2)} \equiv w \\
R_{14}=w-x \quad, \quad R_{31}^{(1)}=R_{42} \quad=x+y-w \quad, \quad R_{31}^{(2)}=x+z \\
R_{23}^{(1)}=R_{15}=2-x-y-z \quad, \quad R_{34}=2-x-y \quad .
\end{aligned}
$$

\footnotetext{
${ }^{10} \mathrm{As}$ in the previous Section we will assume that the nodes of the quiver will all be interacting in the IR where the theory will reach a non-trivial fixed point.

${ }^{11} \mathrm{As}$ for $d P_{1}$, in the $a$-maximization calculations present in the literature for the $d P_{2}$ dual, it is always assumed that the $R$-charges of the two doublets are the same, i.e. the flavor $S U(2) \times S U(2)$ is unbroken. This leads to the conclusion that the $R$-charges and the central charge (and hence the volumes of the dual geometry) are rational.
} 
Plugging these values in Eq. (1.2) we get for the trial $a$-charge

$$
\begin{aligned}
a_{t}= & \frac{9 N^{2}}{32}\left[5+(x-1)^{3}+(y-1)^{3}+(z-1)^{3}+(w-1)^{3}+(w-x-1)^{3}+\right. \\
& \left.(x+z-1)^{3}+2(x+y-w-1)^{3}+(1-x-y)^{3}+2(1-x-y-z)^{3}\right]
\end{aligned}
$$

Extremizing with respect to $x, y, w$ and $z$, we get

$$
\begin{array}{rlrl}
x_{m} & =\frac{1}{2}(-5+\sqrt{33}), y_{m} & =\frac{1}{4}(9-\sqrt{33}), \\
w_{m} & =\frac{1}{16}(17-\sqrt{33}) \quad, \quad z_{m}=\frac{3}{16}(19-3 \sqrt{33}),
\end{array}
$$

and hence for the actual central charge of the theory

$$
c=a=\frac{243 N^{2}}{1024}(-59+11 \sqrt{33}) .
$$

Note that, as for $d P_{1}$, the central charge is irrational. It is larger than the one calculated previously in literature, i.e. $27 N^{2} / 28$. Also it predicts, for the volume of the dual compact space, the value

$$
V\left(X_{d P_{2}}\right)=\frac{\pi^{3} N^{2}}{4 a}=\frac{1}{486}(59+11 \sqrt{33}) \pi^{3},
$$

where we denote as $X_{d P_{2}}$ the horizon of the complex cone over $d P_{2} \cdot{ }^{12}$

The corresponding $R$-charges for the chiral fields can be now easily computed plugging the values (3.27) in the relations (3.25) and are listed in Table 2. Again, they turn out

\begin{tabular}{|c|c|}
\hline$X_{i j}$ & $R_{i j}$ \\
\hline$X_{23}^{1}, X_{31}^{1}, X_{42}, X_{15}$ & $\frac{1}{16}(-21+5 \sqrt{33})$ \\
$X_{23}^{2}, X_{31}^{2}$ & $\frac{1}{16}(17-\sqrt{33})$ \\
$X_{52}, X_{14}$ & $\frac{3}{16}(19-3 \sqrt{33})$ \\
$X_{53}, X_{34}$ & $\frac{1}{4}(9-\sqrt{33})$ \\
$X_{45}$ & $\frac{1}{2}(-5+\sqrt{33})$ \\
\hline
\end{tabular}

Table 2: The exact $R$-charges of the bi-fundamental chiral fields for the $d P_{2}$ dual, Model II.

to be irrational. It can be checked that with the above $R$-charge assignment there are no violations to the unitarity bound.

\footnotetext{
${ }^{12}$ The above volume is smaller than the one for the $d P_{1}$ case. For the case of regular $d P_{k}$ surfaces it is also expected that the related volumes decrease with $k$ [11].
} 
By using once again the holographic relation (1.5) adapted to this case, we can predict the volumes of the supersymmetric three-cycles of the dual geometry. Using Eq. (3.29) and Table 2, these turn out to be, again, irrational.

As far as Model I is concerned, this is Seiberg dual to Model II, so the physical quantities in the IR such as the central charge should be the same. One can repeat the calculations above and check that this is indeed the case. This is a non-trivial check, since the two theories look pretty different. As expected, the equality holds for $a_{t}(\max )$. Finally, the $R$-charges of the chiral fields are again irrational and are listed in Table 3.

\begin{tabular}{|c|c|}
\hline$X_{i j}$ & $R_{i j}$ \\
\hline$X_{32}, X_{15}^{2}, X_{31}, X_{25}^{2}$ & $\frac{1}{16}(-21+5 \sqrt{33})$ \\
$X_{53}^{1}, X_{53}^{2}$ & $\frac{1}{4}(9-\sqrt{33})$ \\
$X_{41}, X_{42}$ & $\frac{3}{16}(19-3 \sqrt{33})$ \\
$X_{15}^{1}, X_{25}^{1}$ & $\frac{1}{16}(17-\sqrt{33})$ \\
$X_{34}$ & $\frac{1}{2}(-5+\sqrt{33})$ \\
$X_{54}$ & $\frac{1}{8}(-21+5 \sqrt{33})$ \\
$X_{53}^{3}$ & $\frac{1}{8}(37-5 \sqrt{33})$ \\
\hline
\end{tabular}

Table 3: The exact $R$-charges of the bi-fundamental chiral fields for the $d P_{2}$ dual, Model I.

Let us end with a comment. The value of the volume of $X_{d P_{2}}$, Eq. (3.29), is close to the one of $Y^{3,1}$. From the general formula (1.1) this reads

$$
V\left(Y^{3,1}\right)=\frac{1}{648}(63+11 \sqrt{33}) \pi^{3} .
$$

This is smaller than $V\left(X_{d P_{2}}\right)$, and thus $a\left(X_{d P_{2}}\right)<a\left(Y^{3,1}\right)$. The SCFT dual to $Y^{3,1}$ has $S U(N)^{6}$ gauge group [6], while for the dual of $X_{d P_{2}}$ this is $S U(N)^{5}$. What one can argue is that the two SCFT's may be related by some higgsing. It would be clearly interesting to find an explicit realization of this higgsing and a metric for $X_{d P_{2}}$.

\section{Acknowledgments}

We thank B. Acharya, D. Martelli, J. Sparks and Y. Takanishi for discussions and exchange of ideas. We are grateful to K. Intriligator for very interesting remarks and e-mail correspondence and to A. Schwimmer for useful comments. We finally thank the authors of [6] for communicating their geometric findings prior to publication, and for earlier collaboration on related material. Work partially supported by the European Commission 
RTN Program MRTN-CT-2004-005104, MRTN-CT-2004-503369, EC Excellence Grant MEXT-CT-2003-509661 and by MIUR. M.B. is also supported by a MIUR fellowship within the program "Incentivazione alla mobilità di studiosi stranieri e italiani residenti all'estero".

\section{References}

[1] J. M. Maldacena, The large $N$ limit of superconformal field theories and supergravity, Adv. Theor. Math. Phys. 2 (1998) 231 [Int. J. Theor. Phys. 38 (1999) 1113], hep-th/9711200. S.S. Gubser, I.R. Klebanov, A.M. Polyakov, Gauge Theory Correlators from Non-Critical String Theory, Phys. Lett. B 428 (1998) 105, hep-th/9802109. E. Witten, Anti De Sitter Space And Holography, Adv. Theor. Math. Phys. 2 (1998) 253, hep-th/9802150.

[2] A. Kehagias, New Type IIB Vacua and their F-Theory Interpretation, Phys. Lett. B 435 (1998) 337, hep-th/9805131. B. S. Acharya, J. M. Figueroa- O'Farrill, C. M. Hull, B. Spence, Branes at conical singularities and holography, Adv. Theor. Math. Phys. 2 (1999) 1249, hep-th/9808014. D. R. Morrison, M. R. Plesser, Non-Spherical Horizons, I, Adv. Theor. Math. Phys. 3 (1999) 1, hep-th/9810201.

[3] I. R. Klebanov, E. Witten, Superconformal Field Theory on Threebranes at a CalabiYau Singularity, Nucl. Phys. B 536 (1998) 199, hep-th/9807080.

[4] J. P. Gauntlett, D. Martelli, J. Sparks, D. Waldram, Sasaki-Einstein Metrics on $S^{2} \times S^{3}$, hep-th/0403002.

[5] M. Henningson, K. Skenderis, The Holographic Weyl anomaly, JHEP 07 (1998) 023, hep-th/9806087.

[6] D. Martelli, J. Sparks, Toric Geometry, Sasaki-Einstein Manifolds and a new Infinite Class of AdS/CFT duals, hep-th/0411238

[7] B. Feng, A. Hanany, Y. H. He, D-brane gauge theories from toric singularities and toric duality, Nucl. Phys. B 595 (2001) 165, hep-th/0003085.

[8] E. Witten, Baryons and Branes in Anti-de Sitter Space, JHEP 07 (1998) 6, hep-th/9805112 
[9] D. Berenstein, C.P. Herzog, I.K. Klebanov, Baryon Spectra and AdS/CFT correspondence, JHEP 06 (2002) 047, hep-th/0202150.

[10] K. Intriligator, B. Wecht, The exact superconformal R-symmetry maximizes a, Nucl. Phys. B 667 (2003) 183, hep-th/0304128.

[11] K. Intriligator, B. Wecht, Baryon charges in 4D superconformal field theories and their AdS duals, Commun. Math. Phys. 245 (2004) 407, hep-th/0305046.

[12] D. Kutasov, A. Parnachev, D.A. Sahakyan, Central Charges and $U(1)_{R}$ Symmetries in $\mathcal{N}=1$ Super Yang-Mills, JHEP 0311 (2003) 013, hep-th/0308071.

[13] D. Kutasov, New results on the "a-theorem" in Four Dimensional Supersymmetric Field Theory, hep-th/0312098.

[14] E. Barnes, K. Intriligator, B. Wecht, J. Wright, Evidence for the Strongest Version of the $4 d$ a-Theorem via a-Maximization Along RG Flows, Nucl. Phys. B 702 (2004) 131, hep-th/0408156.

[15] D. Kutasov, A. Schwimmer, Lagrangian Multipliers and Couplings in Supersymmetric Field Theory, Nucl. Phys. B 702 (2004) 369, hep-th/0409029.

[16] C. P. Herzog, J. McKernan, Dibaryon spectroscopy, JHEP 0308 (2003) 054, hep-th/0305048.

[17] S. Franco, A. Hanany, Y. H. He, P. Kazakopoulos, Duality walls, duality trees and fractional branes, hep-th/0306092.

[18] S. Franco, Y. H. He, C. Herzog, J. Walcher, Chaotic Duality in String Theory, Phys. Rev. D 70 (2004) 046006, hep-th/0402120.

[19] I.R. Klebanov, A.A. Tseytlin, Gravity Duals of Supersymmetric $S U(N) x S U(N+M)$ Gauge Theories, Nucl. Phys. B 578 (2000) 123, hep-th/0002159.

[20] I.R. Klebanov, M. Strassler, Supergravity and a Confining Gauge Theory: Duality Cascades and $\chi S B$-Resolution of Naked Singularities, JHEP 08 (2000) 52, hep-th/0007191.

[21] M. Bertolini, F. Bigazzi, A.L. Cotrone, work in progress. 
[22] A. Ceresole, G. Dall'Agata, R. D'Auria, S. Ferrara, Superconformal field theories from IIB spectroscopy on $A d S_{5} \times T^{11}$, Class. Quant. Grav. 17 (2000) 1017, hep-th/9910066.

[23] B. Feng, S. Franco, A. Hanany, Y. H. He, Symmetries of toric duality, JHEP 12 (2002) 076, hep-th/0205144.

[24] S.S. Gubser, Einstein Manifolds and Conformal Field Theories, Phys. Rev. D 59 (1999) 025006, hep-th/9807164.

[25] G. Tian, S.T. Yau, Kähler-Einstein metrics on compact surfaces with $c_{1}>0$, Commun. Math. Phys. 112 (1987) 175.

[26] A. Bergman, C.P. Herzog, The Volume of some Non-spherical Horizons and the AdS/CFT Correspondence, JHEP 12 (2002) 030, hep-th/0108020. 